\title{
Population Pharmacokinetics of Etomidate Isnotchanged in Patients With Obstructive Jaundice: An Observational Study
}

Jinchao Song

shanghai shidong hospital

Xiaoyan Meng

Eastern Hepatobiliary Surgery Hospital

Hua Yang

Shanghai Shidong Hospital

Hao Gao

Eastern Hepatobiliary Surgery Hospital

Meihua Cai

shanghai children's medical center

Mazhong Zhang

shanghai children's medical center

Weifeng Yu ( $\nabla$ ywf808@sohu.com )

Shanghai Jiao Tong University School of Medicine Affiliated Renji Hospital

Research

Keywords: etomidate, population pharmacokinetics, obstructive jaundice

Posted Date: June 29th, 2020

DOl: https://doi.org/10.21203/rs.3.rs-38059/v1

License: (c) (i) This work is licensed under a Creative Commons Attribution 4.0 International License.

Read Full License 


\section{Title page}

\section{Population Pharmacokinetics of Etomidate isnotchangedin Patients with}

\section{Obstructive Jaundice: an observational study}

Jin-Chao Song, M.D. ${ }^{1,2 *}$, Xiao-yan Meng, M.D. ${ }^{2, *}$, Hua Yang, M.D. ${ }^{1}$, Hao Gao, M.D. ${ }^{2}$, Mei-hua Cai, M.D. ${ }^{3}$, Ma-Zhong Zhang, M.D. ${ }^{3, \#}$, Wei-feng Yu, M.D.,"\#

${ }^{1}$ Department of Anesthesiology, ShidongHospital of Shanghai, University of Shanghai for Science and Technology, 999 Shiguang Road, Shanghai, China

${ }^{2}$ Department of Anesthesiology, Eastern Hepatobiliary Surgery Hospital, the Second Military Medical University, 225 Changhai Road, Shanghai, China.

${ }^{3}$ Department of Anesthesiology, Shanghai Children's Medical Center, Shanghai Jiao Tong University School of Medicine, 1678 Dongfang Road, Shanghai, China.

${ }^{4}$ Department of Anesthesiology, Ren Ji Hospital, School of Medicine, Shanghai Jiao Tong University, 160 Pudian Road, Shanghai, China.

\#Corresponding Author: Wei-Feng Yu, M.D., Professor, Department of Anesthesiology, Ren Ji Hospital, School of Medicine, Shanghai Jiao Tong University, 160 Pudian Road, Shanghai, China; Department of Anesthesiology, Eastern Hepatobiliary Surgery Hospital, the Second Military Medical University, 225 Changhai Road, Shanghai, China. Phone: (86) 21-68383204; Email:ywf808@yeah.net. Ma-Zhong Zhang,M.D., Professor, Department of Anesthesiology, Shanghai Children's Medical Center, Shanghai Jiao Tong University School of Medicine, 1678 Dongfang Road, Shanghai, China; Phone: (86) 21-875230;

Email:CL1236987@126.com. 
*: these authors contribute equally to this work. 


\section{Abstract}

Background: Etomidate is a commonly used in induction of anesthesia. We have previously confirmed that etomidate requirements are significantly reduced in patients with obstructive jaundice and that etomidate anesthesia during ERCP results in more stable hemodynamics when compared with propofol. The aim of the present study is to investigate the pharmacokinetics of etomidate in patientswith and without obstructive jaundice.

Method: 18 patients with obstructive jaundice and 12 non-jaundiced patients scheduled for bile duct surgery were enrolled in the study. Etomidate $0.333 \mathrm{mg} / \mathrm{kg}$ was administered by IV bolus for anesthetic induction. Arterial blood samples were drawn before, during, and up to 300 minutes after bolus. Plasma etomidate concentrations were determined using a validated high-performance liquid chromatography tandem mass spectrometry assay. A population nonlinear mixed-effects modeling approach was used to characterize etomidate pharmacokinetics. Thecovariates age, gender, height, weight, body surface area (BSA), body mass index (BMI), lean body mass (LBM), total bilirubin (TBL), alanine aminotransferase(ALT), aspartate aminotransferase(AST), total bile acid (TBA), creatinine(CR), blood urea nitrogen(BUN)were tested for significant effects on parametersusing a multiple forward selection approach. Covariate effects were judged based onchanges in the objective function value (OFV).

Results:A three-compartment disposition model adequately described the pharmacokinetics of etomidate.The model was further improved when height was a covariate of total clearance $\left.\left[\mathrm{Cl}_{1}=1.30+0.0232(\mathrm{HT}-162), \Delta \mathrm{OFV}=-7.33 ; \mathrm{P}<0.01\right)\right]$. The 
introduction of any other covariates, including bilirubin and total bile acids, did not improve the model significantly $(\mathrm{P}>0.01)$. For the height of $162 \mathrm{~cm}$, the final pharmacokinetic parameter valueswere as follows: $\mathrm{V} 1=1.42$ (95\% CI, 1.01-1.83, L), $\mathrm{V} 2=5.52(95 \% \mathrm{CI}, 4.07-6.97, \mathrm{~L}), \mathrm{V} 3=63.9(95 \% \mathrm{CI}, 41.95-85.85, \mathrm{~L}), \mathrm{Cl} 1=1.30(95 \% \mathrm{CI}$, $1.19-1.41,1 / \mathrm{min}), \mathrm{Cl} 2=1.21(95 \% \mathrm{CI}, 0.95-1.47, \mathrm{~L} / \mathrm{min})$, and $\mathrm{Cl} 3=0.584(95 \% \mathrm{CI}$, 0.95-1.21, L/min), respectively, respectively.

Conclusion: A 3-compartment open model might best describe the concentration profile of etomidate after bolus infusion for anesthesia induction. The pharmacokinetics of etomidate has not been changed in the obstructive jaundice.

Trial registration:The trial was registered prior to patient enrollment at clinicaltrials.gov (ChiCTR-IPR-15007574, Principal investigator: Jin-chao Song, Date of registration: 2015-12-10).

Key words: etomidate; population pharmacokinetics; obstructive jaundice 


\section{Background}

Etomidate is a commonly used ininduction of anesthesia. The mechanism of etomidate's hypnotic effect involves binding to $\gamma$ aminobutyric acid (GABA)type A receptors in the central nerve system and potentiating the effect of GABA[1, 2]. In healthy volunteers, etomidate is approximately $76 \%$ protein bound and has a high solubility in fat[2]. Thus, etomidate is characterized by a large apparent volume of distribution. The major metabolic pathway of etomidate is hydrolysis by carboxylesterase in liver and plasma, with a total clearance rate of $15-20 \mathrm{ml} / \mathrm{kg} / \mathrm{min}$ [3]. There aresignificant alterations in pharmacokinetic parametersof etomidate in the cirrhotic patients[4].We have confirmed that the etomidate requirement in anesthesia induction was significantly reduced in patients with obstructive jaundice[5], and etomidate anesthesia during ERCP is associated withmore stable haemodynamic responses compared with propofol[6].However, pharmacokinetics of etomidate inpatients with obstructive jaundice have not been determined. We hypothesized that the pharmacokinetics of etomidatewould be affected by obstructive jaundice. This study was designed to investigatethe etomidate pharmacokinetics in patients with and without obstructive jaundice.

\section{Materials and Methods}

\section{Clinical trail}

This study was approved by the Ethics Committee of Eastern Hepatobiliary Surgery Hospital (EHBHKY2013-002-003), and written informed consent was obtained from 
all subjects participating in the trial. The trial was registered prior to patient enrollment at clinicaltrials.gov (ChiCTR-IPR-15007574, Principal investigator: Jin-chao Song, Date of registration: 2015-12-10).Participants were patients scheduled for elective bile duct surgery in 2016, were 18 to 70 years of age, and weighed 50 to $80 \mathrm{~kg}$. Exclusion criteria were 1) ASA > 3, 2) severe cardiac, pulmonary, renal, or metabolic disease, 3) analgesic or neuromodulating medication in the past month. A total of 30 patients were recruited into this study. Eighteen of them had obstructive jaundice secondary to malignant disease. Twelve patients without jaundice serviced as a control group. Written informed consent were obtained from each individual prior to the surgery.

The patients were fasted overnight. A radial artery catheter (20G, BL BRUN B.) was also inserted under local anesthesia for invasive monitoring of blood pressure and blood sampling, and a cannula (CV-501-20, Central Venous Catheter, Ltd, Singapore) was inserted into an internal jugular vein for drug administration, fluid infusion and CVP measuring. Other monitors including: noninvasive blood pressure, heart rate, ECG, pulse oximetry, and end-tidal carbon dioxide continuously throughout the surgery. Etomidate $0.333 \mathrm{mg} / \mathrm{kg}$ was injected over 20 seconds, sufentanil $(20-30 \mu \mathrm{g})$ and muscle relaxation rocuronium $(50-75 \mathrm{mg})$ were injected 5 minutes later for anesthesia induction. After tracheal intubation, anesthesia was maintained with 2-4\% sevoflurane in oxygen and by bolus administration of sufentanil $(10 \mu \mathrm{g})$ and rocuronium $(25-50 \mathrm{mg})$ as required throughout the procedure.

Arterial blood samples $(2 \mathrm{ml})$ were drawn: before the anesthesia; immediately after etomidate injection, at 1, 2, 3, 5, 10, 15, 20, 30, 60, 90, 120, 150, 180, 240 and $300 \mathrm{~min}$ 
after etomidate administration. Blood samples were collected in EDTA tubes (containing lithium heparin) and were kept on ice. The samples were pre-processed within 15 minutesand stored at $-80^{\circ} \mathrm{C}$ until analysis. The etomidate concentration was determined using high-performance liquid chromatography (Aglient 1200; Aglient, Santa Clara, CA) and a 1100 II diode array detector, as previously described[7, 8].

Pharmacokinetic Analysis

The pharmacokinetic analysis was performed using nonlinear mixed-effect population model (NONMEM) (6).Models were run using the first-order conditional estimation with interaction method, one-, 2- or 3-compartment models were investigated. Models were parameterized by apparent central volume of distribution (V1, L); peripheral volume of distribution (V2, V3, L); total clearance (CL1, mL/min), intercompartmental clearance (CL2, CL3, $\mathrm{mL} / \mathrm{min})$.

The variability of population pharmacokinetic parameters across individuals were described as $\theta_{i}=\theta_{T} \cdot e^{\eta_{i}}$, where $\theta_{i}$ is the estimated parameter value for the individual subject $i, \theta_{T}$ is the typical population value of $\mathrm{PK}$ parameter $\mathrm{T}$, $\eta \mathrm{i}$ are the interindividual random effects for individual. Random residual variability was described $\operatorname{as} \log \left(y_{i j}\right)=\log \left(\hat{y}_{i j}\right)+\varepsilon_{i j}$, where $y_{i j}$ is the observed concentration in individual $\mathrm{i}$ on measurement time $\mathrm{j}, \hat{y}_{i j}$ is the individual-predicted concentration, while $\varepsilon_{i j}$ is the residual random error.

Once the base model was constructed, the available covariates, including age, gender, height, weight, body surface area (BSA), body mass index (BMI), lean body mass (LBM), total bilirubin (TBL), alanine aminotransferase (ALT), aspartate 
aminotransferase (AST), total bile acid (TBA), creatinine (CR), blood urea nitrogen (BUN) were estimated using multiple forward selection to define the covariate effects on model parameter. Covariates were considered significant when the difference between the objective function values with and without the covariate was greater than $6.63(\mathrm{P}<0.01)[9,10]$. The goodness-of-fit from each NONMEM run was assessed by the examination and presented in visual inspection of diagnostic scatter plots: observed concentration versus individual- and population-predicted concentration; residuals (RES) and weight residuals (WRES) vs. time and population predicted concentrations; observed concentration versus individual- and population-predicted concentration in each subject[9].

Statistical analysis

Measurement data are expressed as mean \pm standard deviation (SD). Statistical analysis was analyzed with independent 2-sample t-test (2-sided) or non-parametric test. The count data were compared using chi-square. $\mathrm{P}<0.05$ at two tails was considered statistically significant. Analyses and figures were made using SPSS 24.0 (SPSS Inc., Chicago, IL) and S-Plus 8.0.4 (Insightful Corp., Seattle, WA, USA).

\section{Results}

The demographic details of the two groups are presented in Table 1, obstructive jaundice patients have higher value of TBL and ALT, no significant difference exist in other variables. A total of 418 blood samples from 30 patients were successfully collected and analyzed. The concentration-time profiles are presented in Figure 1. 
The NONMEM model is constructed based on the data from the 30 individuals. A 3-compartment disposition model (with a lowest objective function value of -434.732 , run1.1-1.3, Supplementary table 1) is deemed optimal to define the etomidate plasma concentration profile based on results from the model-building process and previously published data. By applying all the variates into the model, a full covariate model is ultimately constructed with all parameter estimates and interindividual variability are presented in Table 2aand $2 \mathrm{~b}$. Height is evaluated as a covariate on CL (result in significant improvement in the objective function of model fitting, run 17.5, Supplementary table 1). No significant effect on CL have been found in other variates, including TBL, TBA, ALT and AST.

The systematic bias in the estimation of plasma concentrations for the model is estimated ultimately. Figure 2 presents the measured concentrations/population predicted concentrations and measured concentrations/individual predicted concentrations using post-hoc prediction, Figure 3 presents the WRES and RES vs. time and population predicted concentrations, and Figure 4 shows a plot of the observed, population-predicted, and individual predicted concentrations versus time for the full covariate model. All these results suggest promising prediction value with minimal systematic bias of the final model.

\section{Discussion}

The present study investigates the pharmacokinetics of etomidate in patients undergoing bile duct surgery, and analyzes the effect of increased bilirubin on 
etomidate distribution and metabolism. The pharmacokinetics of etomidate were described by a three-compartment model. The calculated estimate for total clearance rate was $1.30+0.0232(\mathrm{HT}-162) 1 / \mathrm{min}$, volume of the central compartment was $1.42(95 \%$ CI, 1.01-1.83 L), and volumes of the two peripheral compartments were 5.52 (95\% CI, 4.07-6.97 L) and 63.9 (95\% CI, 41.95-85.85 L), respectively. The introduction of height as a covariate for systemic clearance further improved the model and resulted in a significant reduction of OFV. Unexpectedly, when introducing the obstructive jaundice associated variables, including bilirubin, TBA, ALT and AST, none of them have significant effect on the final model.Therefore, we may conclude that the etomidate pharmacokinetics in patients with obstructive jaundice are similar to those of patients without obstructive jaundice.

Etomidate was firstly introduced into clinical practice in 1972, and academicpublications focusing on its' pharmacokinetics/pharmacodynamics rose soon afterward[11]. An initial research had reported that a 3-compartment open pharmacokinetic model might fit for the plasma concentration profile of a single intravenous injection of etomidate. Further, etomidate is also characterized by a large central volume of distribution $(4.5 \pm 2.2 \mathrm{~L})$, even larger peripheral volume of distribution, a short distribution and elimination half-life $(2.6 \pm 1.3 \mathrm{~min}$ and $4.6 \pm 2.6 \mathrm{hr}$, respectively), and a total plasma clearance of $860 \pm 230 \mathrm{ml} / \mathrm{min}[12]$. Whereafter, more researches reported that the pharmacokinetic distribution of etomidate conforms to the three-compartment open model. Ruiter et al.[13] built a pharmacokinetic model based 
on eight adult surgical patients, and following mean parameters were computed: initial distribution phase half-life $(\mathrm{t} 1 / 2 Л)$ is $2.8 \pm 2.3 \mathrm{~min}$, half-life of the alpha-phase of distribution $(\mathrm{t} 1 / 2 \alpha)$ is $22.3 \pm 10.4 \mathrm{~min}$, and half-life of the beta-phase of elimination $(\mathrm{t} 1 / 2 \beta)$ is $208.8 \pm 64.9 \mathrm{~min}$, the total volume of apparent distribution $(\mathrm{Vd})$ is 3.68 or $0.66 \mathrm{~L} / \mathrm{kg}$, the central volume of apparent distribution $(\mathrm{Vc})$ is 2.16 , and the total plasma clearance (CL) is $879 \pm 135 \mathrm{ml} / \mathrm{min}$. Nevertheless, controversy still exist in the pharmacokinetic model of etomidate. In 2011, Kaneda et al.[14] analyzed the population pharmacokinetics and pharmacodynamics of brief etomidate infusion in eighteen healthy volunteers. Their data results in a 2-compartment pharmacokinetic model and a pharmacodynamic sigmoid Emax model for etomidate, the central and peripheral volumes of distribution are $4.45 \mathrm{~L}$ and $74.90 \mathrm{~L}$, respectively, the systemic and intercompartmental clearances are 0.63 and $3.16 \mathrm{~L} / \mathrm{min}$, respectively. Our findings are consistent with mainstream opinion that plasma concentrations of etomidate after single bolus in human best fit a 3-compartment open model. Our results also suggest large apparent volume and quick clearance of etomidate, with a fast, intermediate, and slow declines of concentration in plasma.

Approximately $76 \%$ of etomidate is bound to plasma albumin, mainly hydrolyzed by esterase in the liver and plasma and then metabolized to carboxylic acids. The carboxylate metabolite is excreted mostly in urine (85\%) and bile (13\%). Etomidate has a high liver clearance rate of $18-25 \mathrm{ml} /$ (kg.min), a liver uptake rate of 0.5 to $0.9[15]$, and its metabolic clearance is approximately equal to the hepatic blood flow. Of note, numerous evidences have identified altered hepatic blood flow in obstructive jaundice. 
Kands et al.[16] used a real-time ultrasound flow meter to measure portal blood flow and hepatic arterial blood flow in dogs with obstructive jaundice, and found that the hepatic arterial blood flow was significantly higher than that before obstruction while the portal vein blood flow was significantly lower. Meanwhile, Kulibaba et al. also identified a compensatory increase of blood flow in the hepatic artery in obstructive jaundice patients[17]. Further, Bonnardot et al.[4] performed a prospective clinical study, and found significant alterations in pharmacokinetic parameters in the cirrhotic patients. Based on these findings, we hypothesized that changes in liver hemodynamics and liver microcirculation caused by obstructive jaundice could result in alterations in the pharmacokinetic characteristics of etomidate. However, our results failed to confirm this, in that the variables of bilirubin level had no significant effect on the clearance rate in the final model. The reason might be that changes in fluid dynamics and liver microcirculation in obstructive jaundice are insufficient to affect the etomidate clearance.

Several limitations of this study should be mentioned. First of all,our study may have an unclear risk of bias due to the small sample sizeof a rather homogenous population with complicated physical conditions. Therefore, any relationship with the covariates might be difficult to discern.Second, the covariates of age, weight as well as obstructive associated factors provided no improvement in the objective function of the model, while height significantly affects the estimated value of systematic clearance rate. This is an interesting result as previous evidence suggested that height also affects the pharmacokinetic model of other sedative anesthetics[18, 19]. However, current data are 
insufficient to verify and explain this phenomenon.It is clearthat the current study will provide a framework to design future studies. Third, considering the nature of this clinical research, it is hard to explain or identify whether there are pharmacokinetic interactions between etomidate and other drugs used during surgery. Although etomidate produced no serious adverse events in our patients, the current study is neither intended to nor sufficiently powered to evaluate safety for etomidate in anesthesia induction, therefore, conclusions regarding safety cannot be made.

In conclusion, in this clinical study, we investigated the population pharmacokinetics of etomidatebased on 30 surgical patients with or without obstructive jaundice. In consistent with most studies, we identified that a 3-compartment open model might best describe the concentration profile of etomidate after bolus infusion for anesthesia induction. What's more, we found that the accumulation of bilirubin or bile acids in obstructive jaundice does not appear to affect etomidate pharmacokinetics.

\section{List of abbreviations}

ALT:alanine aminotransferase,AST: aspartate aminotransferase, GABA: $\gamma$ aminobutyric acid,NONMEM:nonlinear mixed-effect population model, BMI: body mass index,BSA: body surface area, BUN:blood urea nitrogen,CR:creatinine,CL:plasma clearance,LBM: lean body mass, TBL: total bilirubin, TBA: total bile acid, OFV:objective function value, RES: residuals, Vd: apparent distribution, Vc: central volume of apparent distribution,WRES:weight residuals. 


\section{Ethics approval and consent to participate}

This study was approved by the Ethics Committee of Eastern Hepatobiliary Surgery Hospital (EHBHKY2013-002-003), and written informed consent was obtained from all subjects participating in the trial.

\section{Consent for publication}

Not applicable.

\section{Availability of data and materials}

The datasets used and/or analysed during the current study are available from the corresponding author on reasonable request.

\section{Competing interests}

The authors declare that they have no competing interests.

\section{Funding}

The Survey was funded by the National Natural Science Foundation of China (grants 81371511, 81270414 and 81202597) and Shanghai Municipal Commission of Health and Family Planning, Key Developing Disciplines (2015ZB0106).

\section{Authors' contributions}

Dr. JC Song performed the study and analyzed the data; XY Meng analyzed the data and wrote the manuscript; H Yang, H Gao, MH Cai performed the study; MZ Zhang designed the study and revised the manuscript, WF Yu designed the study and revised the manuscript.All authors read and approved the final manuscript.

\section{Acknowledgements}

Not applicable. 


\section{Reference}

1. Forman SA, Ph. D: Clinical and Molecular Pharmacology of Etomidate.

2. Giese JL, Stanley TH: Etomidate: A New Intravenous Anesthetic Induction Agent. Pharmacotherapy the Journal of Human Pharmacology \& Drug Therapy 1983, 3(5).

3. Amend DF, Goven BA, Elliot DG: Etomidate: Effective Dosages for a New Fish Anesthetic. Transactions of the American Fisheries Society, 111(3):337-341.

4. Bonnardot JP, Levron JC, Deslauriers M, Brule ML, Deligne P: [Pharmacokinetics of continuous infusion of etomidate in cirrhotic patients]. Annales Françaises Danesthèsie Et De Rèanimation 1991, 10(5):443-449.

5. Song JC, Sun YM, Zhang MZ, Yang LQ, Tao TZ, Yu WF: The Etomidate Requirement Is Decreased in Patients with Obstructive Jaundice. Anesthesia \& Analgesia, 113(5):1028-1032.

6. Song JC, Zhi-Jie L, Ying-Fu J, Bin Y, Hao G, Jinmin Z, Wei-Feng Y: Etomidate Anesthesia during ERCP Caused More Stable Haemodynamic Responses Compared with Propofol: A Randomized Clinical Trial. International Journal of Medical Sciences, 12(7):559-565.

7. Jin-Chao, Song, Hao, Gao, Hai-Bo, Qiu, Qian-Bo, Chen, Mei-Hua, Cai: The pharmacokinetics of dexmedetomidine in patients with obstructive jaundice: $A$ clinical trial.

8. Song J, Sun Y, Zhang M, Yang L, Song J, Wang Z, Yu W: Propofol Pharmacokinetics in Patients with Obstructive Jaundice. Current Drug Delivery, 6(3):317-320.

9. Ribbing J, Jonsson EN: Power, Selection Bias and Predictive Performance of the Population Pharmacokinetic Covariate Model.

10. Walker, Esteban: Regression Modeling Strategies. Technometrics, 45(2):170-170.

11. Allen $\mathrm{C}$, Washington $\mathrm{S}$ : The role of etomidate as an anasthetic induction agent for critically ill patients. BrJ Hosp Med, 77(5):282-286.

12. Van Hamme MJ, Ghoneim MM, Ambre JJ: Pharmacokinetics of Etomidate, a New Intravenous Anesthetic. Anesthesiology, 49(4):274-277.

13. De RG, Popescu DT, de Boer AG, Smeekens JB, Breimer DD: Pharmacokinetics of etomidate in surgical patients. 1981, 249(2):180-188.

14. Kaneda K, Yamashita S, Woo S, Han TH: Population Pharmacokinetics and Pharmacodynamics of Brief Etomidate Infusion in Healthy Volunteers. Journal of Clinical Pharmacology 2011, 51(4).

15. Levron JC, Assoune P: Pharmacokinetics of etomidate. Ann Fr Anesth Reanim 1990, 9(2):123-126.

16. Kanda H, Nimura Y, Yasui A, Uematsu T, Shionoya S: Hepatic blood flow after acute biliary obstruction and drainage in conscious dogs. Hepato-gastroenterology 1996 , 43(7):235-240.

17. Kanda H, Nimura Y, Yasui A, Nakano S, Kumada S, Shionoya S: Recovery of Portal Blood Flow After Percutaneous Transhepatic Biliary Drainage in Patients with Obstructive Jaundice. 1997, 27(2):120. 
18. $L L, X G, M Z Z, C J, Q, Y S, J B$ : Pharmacokinetics of dexmedetomidine in Chinese post-surgical intensive care unit patients. Acta anaesthesiologica Scandinavica 2011, 55(3):359-367.

19. JB D, M M, C H, DL A, L V, SL S: Computer-controlled infusion of intravenous dexmedetomidine hydrochloride in adult human volunteers. Anesthesiology 1993, 78(5):821-828. 


\section{Figure legend}

Figure 1. The concentration-time plot for all individuals.

Figure 2. Observed versus individual predicted concentrations (left) and observed versuspopulation predicted concentrations (right).Data are plotted using individual subject identification numbers. A loess smoother is represented by the red line.

Figure 3. Diagnostic scatter plots for the model:Weight residual versus time (A), weight residual versus population predicted concentrations (B), residual versus time (C), residual versus population predicted concentrations (D). $\mathrm{y}=0$ represents good prediction value.

Figure 4.Observed (dot), population(red line), and individualpredicted(blue line) of etomidate concentrations versus time for all individuals. 
Table 1. Demographic Characteristics.

\begin{tabular}{llll}
\hline Variable & Obstructive jaundice $(\mathrm{n}=18)$ & Control $(\mathrm{n}=12)$ & P value \\
\hline Gender male/female & $8 / 10$ & $8 / 4$ & 0.232 \\
Age (years) & $58.9 \pm 7.7$ & $60.1 \pm 4.4$ & 0.657 \\
Height $(\mathrm{cm})$ & $159.9 \pm 6.4$ & $160.7 \pm 5.8$ & 0.989 \\
Weight $(\mathrm{kg})$ & $61.1 \pm 8.0$ & $61.2 \pm 11.6$ & 0.725 \\
Bilirubin $(\mu \mathrm{mol} \cdot \mathrm{L}-1)$ & $109.1 \pm 61.1$ & $10.6 \pm 4.7$ & $\mathbf{0 . 0 0 0}$ \\
ALT (U.L-1) & $156.6 \pm 157.4$ & $24.5 \pm 16.6$ & $\mathbf{0 . 0 1 0}$ \\
AST (U.L-1) & $104.7 \pm 105.7$ & $39.5 \pm 56.9$ & 0.070 \\
Albumin (g.L-1) & $37.9 \pm 3.0$ & $40.1 \pm 5.9$ & 0.192 \\
CR (mmol·L-1) & $62.7 \pm 13.8$ & $62.5 \pm 15.5$ & 0.967 \\
BUN (mmol·L-1) & $5.0 \pm 1.7$ & $5.2 \pm 2.0$ & 0.782 \\
INR & $0.9 \pm 0.1$ & $1.0 \pm 0.1$ & 0.177 \\
\hline
\end{tabular}

$\mathrm{ALT}=$ Alanine aminotransferase; $\mathrm{AST}=$ Aspartate aminotransferase; $\mathrm{CR}=$ creatinine; $\mathrm{BUN}=\mathrm{Blood}$ Urea Nitrogen; INR= international normalized ratio. 
Table 2a. pharmacokinetic variables for each group.

\begin{tabular}{ccccc}
\hline \multirow{2}{*}{ Variable } & Value & \multicolumn{2}{c}{$95 \% \mathrm{CI}$} & \multirow{2}{*}{ CV } \\
\cline { 3 - 4 } & & low & high & \\
\hline Volume & 1.42 & 1.01 & 1.83 & $18.7 \%$ \\
$\mathrm{~V}_{1}(\mathrm{l})$ & 5.52 & 4.07 & 6.97 & $35.8 \%$ \\
$\mathrm{~V}_{2}(\mathrm{l})$ & 63.9 & 41.95 & 85.85 & $48.1 \%$ \\
$\mathrm{~V}_{3}(\mathrm{l})$ & & & & \\
$\mathrm{Clearance}$ & $1.30+0.0232(\mathrm{HT}-162)$ & 1.19 & 1.41 & $17.3 \%$ \\
$\mathrm{Cl}_{1}(1 / \mathrm{min})$ & 1.21 & 0.95 & 1.47 & $23.5 \%$ \\
$\mathrm{Cl}_{2}(1 / \mathrm{min})$ & 0.584 & 0.95 & 1.21 & $42.7 \%$ \\
$\mathrm{Cl}_{3}(1 / \mathrm{min})$ & 0.0232 & 0.0102 & 0.0362 & - \\
\hline $\mathrm{HT}$ on $\mathrm{Cl}_{1}$ & & & & \\
\hline $\mathrm{V}$ & & & & \\
\hline
\end{tabular}

$\mathrm{V}_{1}=$ volume of the central compartment; $\mathrm{V}_{2}, \mathrm{~V}_{3}=$ volumes of the peripheral compartment; $\mathrm{Cl}_{1}=$ total body clearance; $\mathrm{Cl}_{2}, \mathrm{Cl}_{3}=$ Inter-compartmental clearance; $\mathrm{HT}=$ height in centimeter; $\mathrm{CV}=$ Coefficients of variance. All variables based on Nonlinear Mixed Effect Model. 
Table 2b.Other pharmacokinetic variables derived from the model.

\begin{tabular}{lc}
\hline Variables & value \\
\hline Volume of distribution at stead state $(\mathrm{l})$ & 70.84 \\
Coefficient A & 0.957 \\
Coefficient $\mathrm{B}$ & 0.0409 \\
Coefficient C & 0.00156 \\
Index $\left(\mathrm{min}^{-1}\right)$ & \\
$\alpha$ & 2.272 \\
$\beta$ & 0.1294 \\
$\gamma$ & 0.00624 \\
Rate constant $\left(\mathrm{min}^{-1}\right)$ & \\
$\mathrm{K}_{10}$ & 0.915 \\
$\mathrm{~K}_{12}$ & 0.8521 \\
$\mathrm{~K}_{21}$ & 0.2192 \\
$\mathrm{~K}_{13}$ & 0.41127 \\
$\mathrm{~K}_{31}$ & 0.00914 \\
$\mathrm{Half}_{-1 \text { life }(\min )}$ & \\
$\mathrm{t}_{1 / 2} \alpha$ & 0.305 \\
$\mathrm{t}_{1 / 2} \beta$ & 5.4 \\
$\mathrm{t}_{1 / 2} \gamma$ & 111 \\
\hline
\end{tabular}

$\overline{\mathrm{K}_{10}=\text { clearance rate }\left(\mathrm{C}_{1} / \mathrm{V}_{1}\right) ; \mathrm{K}_{12}, \mathrm{~K}_{13}=\text { transportation rate from central to peripheral compartment }}$ $\left(\mathrm{K}_{12}=\mathrm{Cl}_{2} / \mathrm{V}_{1} ; \mathrm{K}_{13}=\mathrm{Cl}_{3} / \mathrm{V}_{3}\right) ; \mathrm{K}_{21}, \mathrm{~K}_{31}=$ transportation rate from peripheral to central compartment $\left(\mathrm{K}_{21}=\mathrm{Cl}_{2} / \mathrm{V}_{2} ; \mathrm{K}_{31}=\mathrm{Cl}_{3} / \mathrm{V}_{3} ;\right) ; \mathrm{t}_{1 / 2} \alpha, \mathrm{t}_{1 / 2} \beta, \mathrm{t}_{1 / 2} \gamma=$ half-times of the three phases. All variables based on Nonlinear Mixed Effect Model, with height set at median value of $162 \mathrm{~cm}$. 
Figures

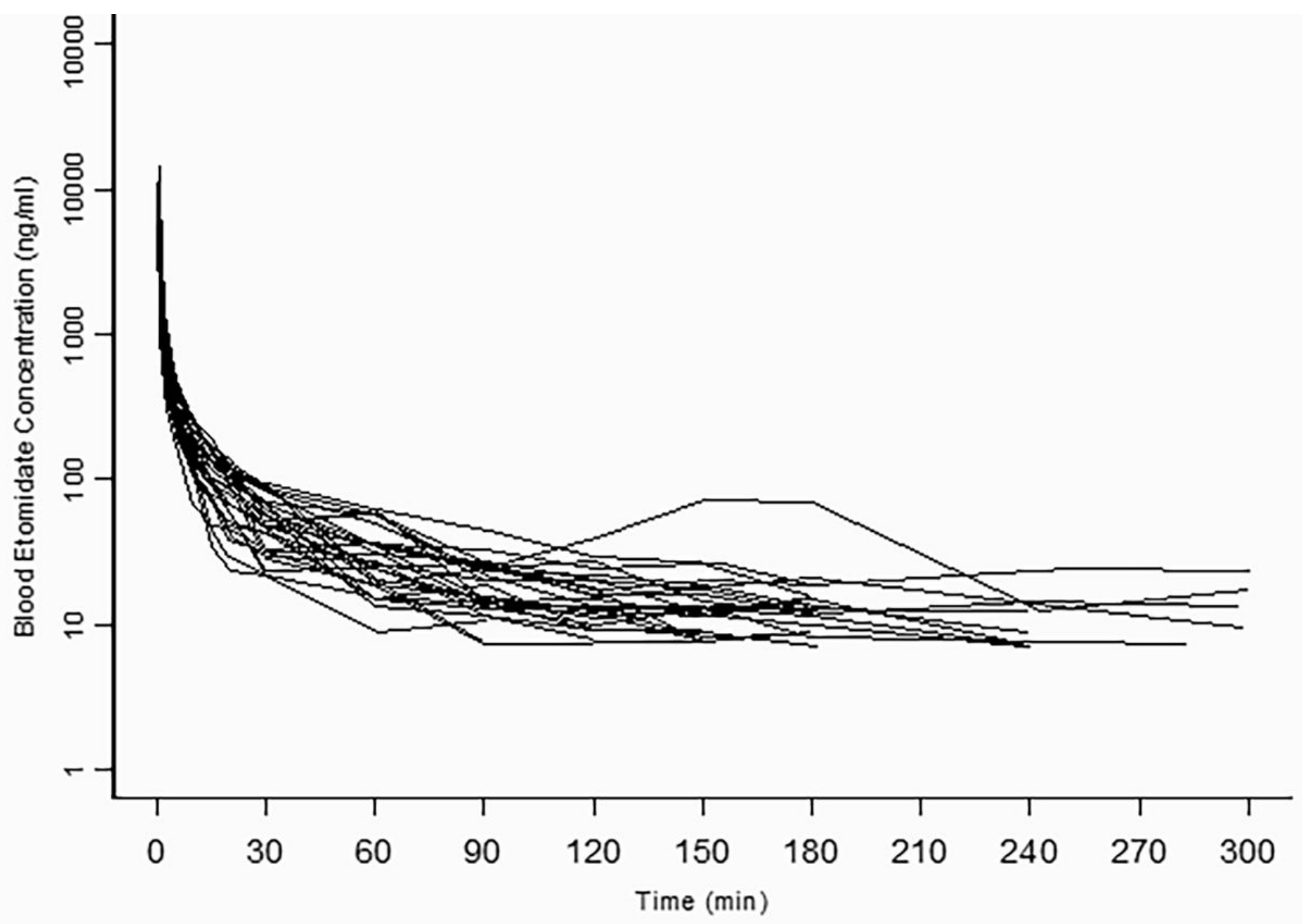

Figure 1

The concentration-time plot for all individuals. 

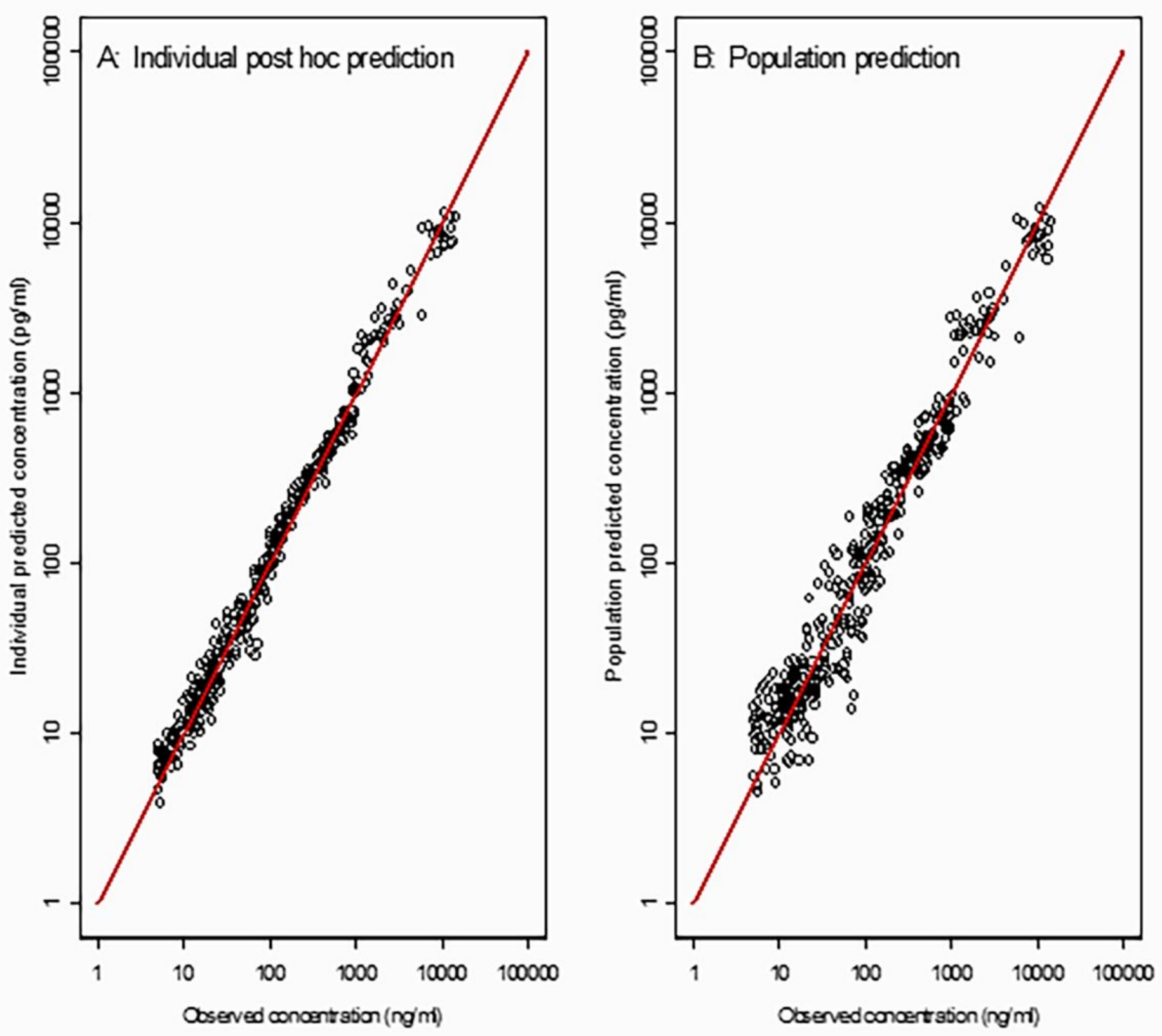

Figure 2

Observed versus individual predicted concentrations (left) and observed versuspopulation predicted concentrations (right).Data are plotted using individual subject identification numbers. A loess smoother is represented by the red line. 

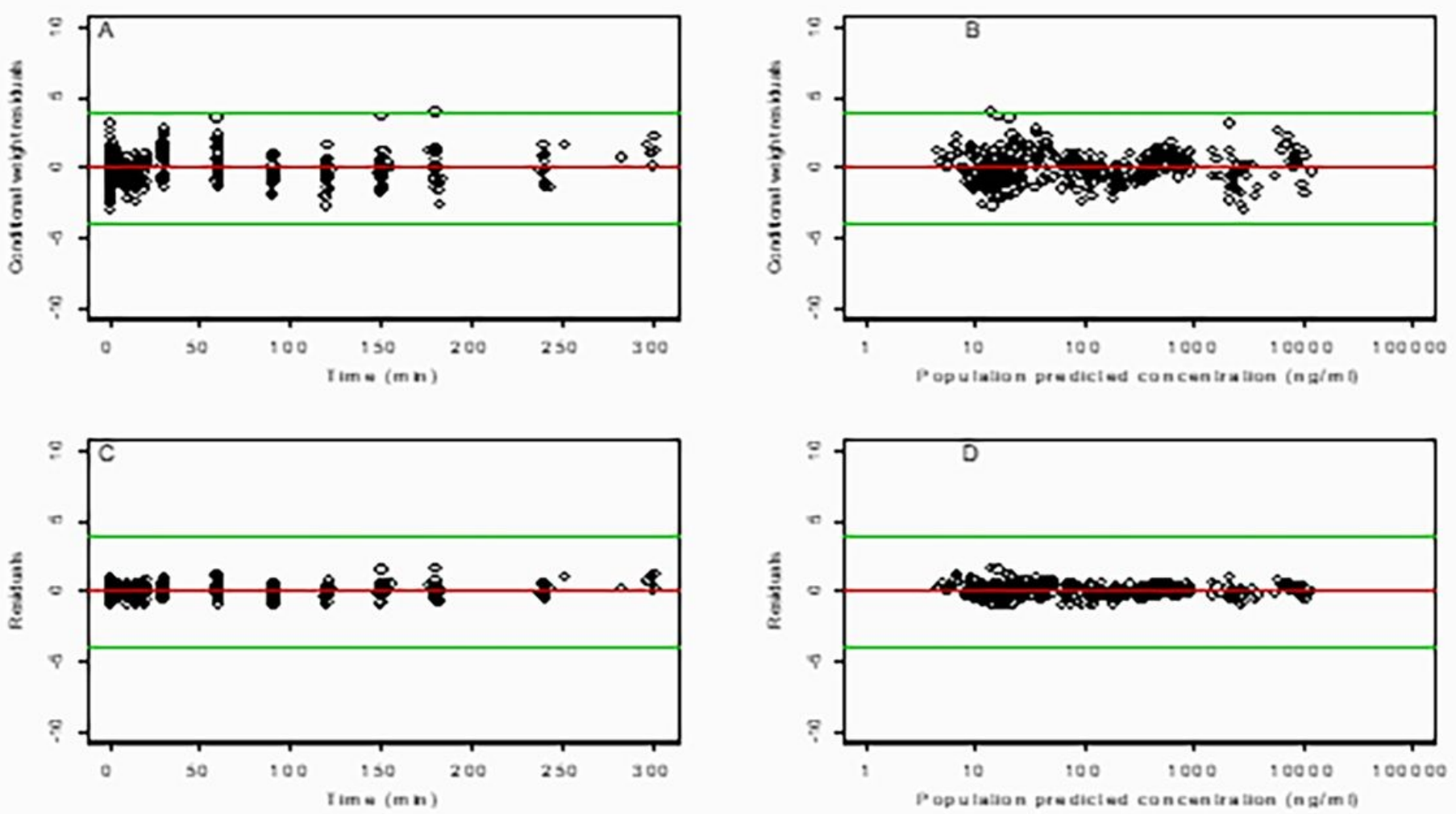

Figure 3

Diagnostic scatter plots for the model:Weight residual versus time (A), weight residual versus population predicted concentrations $(B)$, residual versus time $(C)$, residual versus population predicted concentrations (D). $y=0$ represents good prediction value. 

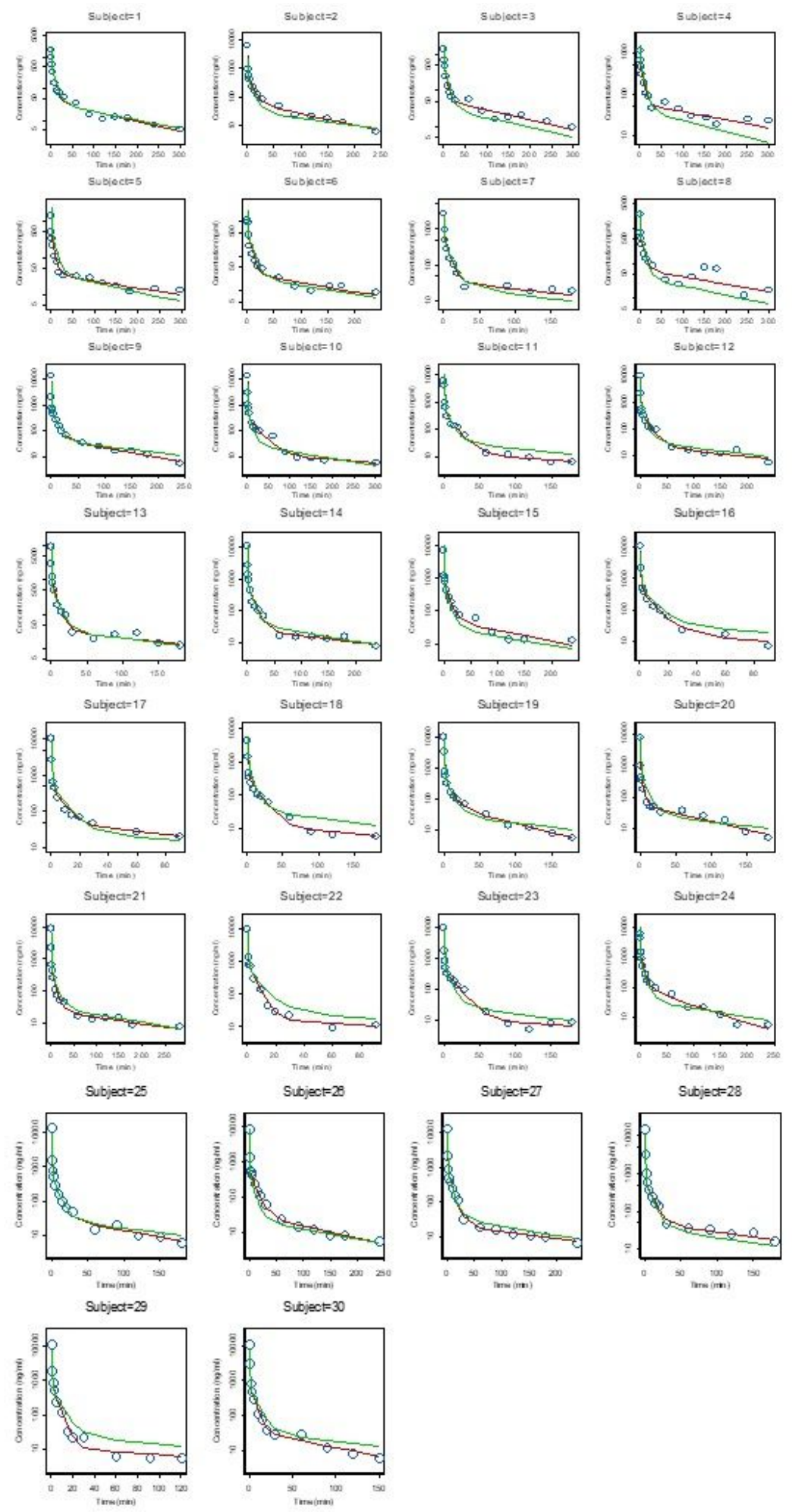

\section{Figure 4}

Observed (dot), population(red line), and individualpredicted(blue line) of etomidate concentrations versus time for all individuals.

\section{Supplementary Files}


This is a list of supplementary files associated with this preprint. Click to download.

- SupplementaryTable1.docx

- SupplementaryTable1.docx 